\title{
Lower Bound on the Magnetic Field Strength of a Magnetar from Analysis of SGR Giant Flares
}

\author{
A. A. Gvozdev, I. S. Ognev † E. V. Osokina \\ Yaroslavl State University, Sovietskaya 14, \\ 150000 Yaroslavl, Russia.
}

\begin{abstract}
Based on the magnetar model, we have studied in detail the processes of neutrino cooling of an electron-positron plasma generating an SGR giant flare and the influence of the magnetar magnetic field on these processes. Electron-positron pair annihilation and synchrotron neutrino emission are shown to make a dominant contribution to the neutrino emissivity of such a plasma. We have calculated the neutrino energy losses from a plasma-filled region at the long tail stage of the SGR 0526-66, SGR 1806-20, and SGR 1900+14 giant flares. This plasma can emit the energy observed in an SGR giant flare only in the presence of a strong magnetic field suppressing its neutrino energy losses. We have obtained a lower bound on the magnetic field strength and showed this value to be higher than the upper limit following from an estimate of the magnetic dipole losses for the magnetars being analyzed in a wide range of magnetar model parameters. Thus, it is problematic to explain the observed energy release at the long tail stage of an SGR giant flare in terms of the magnetar model.
\end{abstract}

Keywords: SGR giant flare, magnetar model, neutrino.

\section{INTRODUCTION}

Soft gamma-ray repeaters (SGRs) and anomalous X-ray pulsars (AXPs) constitute a special class of neutron stars with anomalously large spin periods $P \sim(5-8) s$ and spindown rates $\dot{P} \sim\left(10^{-10}-10^{-13}\right) s s^{-1}$. About 20 such objects have been discovered to date in our and nearest galaxies (Mereghetti 2008). Their identification with supernova remnants shows them to be young isolated neutron stars with ages $\tau_{N S} \sim\left(10^{3}-10^{4}\right)$ yr without accretion disks (Bisnovatyi-Kogan 2006). Note the most characteristic properties of these objects. First, in quiescence they have an anomalously high effective temperature for isolated stars, emitting soft $\mathrm{X}$ rays from the surface with luminosities $L_{N S} \sim\left(10^{33}-\right.$ $10^{36}$ ) (Mereghetti 2008). Second, AXPs exhibit large glitches (Dib et al. 2008), whileSGRs exhibit gamma-ray bursts (Strohmayer and Watts 2006; Watts and Strohmayer 2007). They are interpreted as a manifestation of a seismic activity in these objects similar to the seismicity of the Earth, the Sun, and the young Vela pulsar (Gogus et al. 2000). Third, gamma-ray bursts were also detected from AXPs, although they are not so powerful as

*E-mail: gvozdev@uniyar.ac.ru

${ }^{\dagger}$ E-mail: ognev@uniyar.ac.ru 
those from SGRs (Woods et al. 2005). This suggests that SGRs and AXPs most likely belong to the same class of neutron stars. The SGR flare activity manifests itself in the emission of numerous (up to 100 episodes per day) short bursts in the energy range from hard $\mathrm{X}$ rays to soft gamma rays with a typical duration of $\tau_{F} \sim 0.1$ and energy $E_{F} \lesssim 10^{41}$ erg. In several cases, a series of short bursts was followed by a giant flare exceeding in energetics the short ones by several orders of magnitude. Two easily distinguishable stages were observed in the three most powerful (in energy release) giant flares from SGR 0526-66 (March 5, 1979), SGR 1900+14 (August 27, 1998), and SGR 1806-20 (December $27,2004)$ : a short, with a duration $\tau_{H S} \sim(0.25-0.5)$ and energy $E_{H S} \sim\left(10^{44}-10^{46}\right)$ erg, hard spike (HS) followed by a pulsating long $\left(\tau_{L T} \sim(200-400)\right) s$ tail (LT) with energy $E_{L T} \sim(1-4) \times 10^{44} \mathrm{erg}$, at which a modulation of the emission intensity by the neutron star spin period was observed. Here, we investigate the energy losses at the LT stage of an SGR giant flare and do not consider the HS stage. Below, we present data for the three most energetic SGR giant flares at this stage:

SGR 0526-66 $(D \approx 55 \mathrm{kpc}) \tau_{L T} \approx 200 \mathrm{~s}, E_{L T} \approx 3.6 \times 10^{44}$ erg (Mazets et al. 1979); $\operatorname{SGR} 1900+14(D \approx 15 \mathrm{kpc}) \tau_{L T} \approx 400 \mathrm{~s}, E_{L T} \approx 1.2 \times 10^{44}$ erg (Ibrahim et al. 2001);

SGR $1806-20(D \approx 15 \mathrm{kpc}) \tau_{L T} \approx 380 \mathrm{~s}, E_{L T} \approx 1.3 \times 10^{44} \mathrm{erg}$ (Mereghetti et al. 2005; Frederiks et al. 2007). As can be seen, these parameters almost coincide, suggesting a unified flare formation mechanism at the LT stage.

Note that the energy release in SGR giant flares is smaller than that in supernova explosions and cosmological gamma-ray bursts only. The model of a magnetar, a neutron star with an anomalously strong magnetic field $B_{M} \sim 10^{15} \mathrm{G}$ (Duncan and Thompson 1992; Thompson and Duncan 1993), was proposed to explain such a huge gamma-ray burst energy for an isolated neutron star. In this model, the energy of the magnetic field liberated when its configuration changed rapidly is assumed to be the gamma-ray energy source. Subsequently, the magnetar model was used to describe the emission from AXPs and SGRs in quiescence (Thompson and Duncan 1996) and during an SGR giant flare (Thompson and Duncan 1995). In the magnetar model of an SGR giant flare (Thompson and Duncan 1995), the long-term $\left(\tau_{N S} \sim 10^{3} \mathrm{yr}\right)$ evolution of the magnetar core with poloidal and toroidal magnetic fields of strength $B_{M} \sim 10^{15} \mathrm{G}$ is assumed to be ended with a starquake leading to a largescale plastic deformation of its crust. The electric currents emerging during the deformation produce a perturbative magnetic field with field lines closed on the crust. The region with closed field lines is rapidly (in hundredths of a second) filled with an electron- positron plasma trapped by this field (the so-called fireball). A fairly hot plasma, with a temperature $T \sim 10^{10} \mathrm{~K}$, is generated. The $\mathrm{X}$-ray photon flux observed at the LT stage is assumed to be emitted from a thin nearsurface layer of the fireball. Thompson and Duncan (2001) modeled this emission and compared it with the observed light curve of the SGR 1900+14 giant flare. As a result, the distributions of plasma parameters (temperature and magnetic field) that agreed best with the observational data were obtained. It is important to note that the neutrino emission was disregarded by these authors, because it was assumed to be significantly suppressed by a strong magnetic field inside the fireball.

Here, we study in detail the plasma neutrino emission processes at the LT stage based on the magnetar model of an SGR giant flare. We show that the plasma energy losses through neutrino emission are significant even in the case of a strong magnetic field with a strength $B \gtrsim 10^{15} \mathrm{G}$. For the most energetic flares from SGR 0526-66, SGR 1806-20, and SGR 1900+14, we model the fireball neutrino cooling. The dependences of neutrino cooling on parameters of the temperature and magnetic field distributions, fireball size, and its total energy are analyzed. 
Below, except for the specially stipulated cases, we use a system of units in which $c=\hbar=k=1$.

\section{NEUTRINO COOLING OF A RELATIVISTIC NONDEGENERATE ELECTRON-POSITRON PLASMA}

\section{Main Neutrino Processes}

The following reactions are the most significant neutrino emission processes of a relativistic nondegenerate electron-positron plasma: the electron-positron pair annihilation into a pair of neutrinos with an arbitrary flavor

$$
e^{-}+e^{+} \rightarrow \nu_{i}+\tilde{\nu}_{i}
$$

the plasmon decay into a neutrino pair

$$
\gamma \rightarrow \nu_{i}+\tilde{\nu}_{i}
$$

the neutrino production due to the fusion of two photons

$$
\gamma+\gamma \rightarrow \nu_{i}+\tilde{\nu}_{i}
$$

the photoneutrino emission process

$$
e^{\mp}+\gamma \rightarrow e^{\mp}+\nu_{i}+\tilde{\nu}_{i},
$$

the neutrino synchrotron emission by electrons

$$
e^{\mp} \stackrel{B}{\rightarrow} e^{\mp}+\nu_{i}+\tilde{\nu}_{i}
$$

which is kinematically allowed only in an external magnetic field. Here and below, the subscript $i=e, \mu, \tau$ specifies the neutrino flavor.

\section{The Case of a Weak Magnetic Field}

We will begin our analysis of the neutrino emission processes with the case of an electron-positron plasma in the absence of a magnetic field. The neutrino emissivity of an ultrarelativistic nondegenerate plasma in the annihilation process (1) is well known (Kaminker et al. 1992) and can be written as

$$
Q_{A}^{(0)}=\frac{7 \zeta(5) C_{+}^{2}}{12 \pi} G_{F}^{2} T^{9}
$$

Here, $T$-is the plasma temperature, $G_{F}$-is the Fermi constant, $C_{+}^{2}=\sum_{i}\left(c_{v_{i}}^{2}+c_{a_{i}}^{2}\right) \simeq$ 1.675, where $c_{v_{i}}$ and $c_{a_{i}}$ are the vector and axial constants of the leptonic electroweak current, and $\zeta(x)$ is the Riemann zeta function.

The emissivity in the plasmon decay into a neutrino pair (2) has also been well studied and can be found, for example, in Yakovlev et al. (2001) and Kantor and Gusakov (2007). For a nondegenerate plasma, it can be represented as

$$
Q_{\mathrm{P}}^{(0)}=\frac{C_{v}^{2}}{324 \pi} \alpha^{2} G_{F}^{2} T^{9}
$$


where $\alpha=1 / 137$-is the fine-structure constant and $C_{v}^{2}=\sum_{i} c_{v_{i}}^{2}=0.9248$. It is easy to see that the emissivity in this process is strongly suppressed compared to the electronpositron pair annihilation.

The processes (3) and (44) in a plasma were extensively studied previously (Beaudet et al. 1967; Itoh et al. 1996) and the neutrino emissivities in these processes were shown to be also negligibly small compared to (6). Thus, the annihilation process makes a major contribution to the neutrino emissivity of a relativistic nondegenerate plasma.

In the case of a relatively weak magnetic field, $T^{2} \gg e B$ the emissivities in the processes (1), (2), (3) and (4) change insignificantly, but the presence of a magnetic field makes the new synchrotron neutrino pair production process (5) kinematically open. The emissivity in this process in the limit of a weak magnetic field is given by the expression (Kaminker and Yakovlev 1993):

$$
Q_{S}^{(0)}=\frac{10 \zeta(5)}{9(2 \pi)^{5}} C_{+}^{2} G_{F}^{2}(e B)^{2} T^{5}\left[\ln \left(\frac{T^{2}}{e B}\right)+4.66\right]
$$

from which it follows that $Q_{S}^{(0)} / Q_{A}^{(0)} \sim\left(e B / T^{2}\right)^{2}$ i.e., the emissivity in this process in the limit under consideration is also suppressed. Thus, in a relatively weak magnetic field, the $e^{ \pm}$pair annihilation reaction is the main plasma neutrino cooling process and the total neutrino emissivity is defined by Eq. (6) .

The above analysis allows the characteristic plasma neutrino cooling time $\tau_{\nu}^{(0)}$ to a temperature $T$ to be calculated. It can be found from the equation

$$
\frac{d}{d t}\left[\frac{11 \pi^{2}}{60} T^{4}\right]=-Q_{A}^{(0)}
$$

where the total energy density of an ultrarelativistic nondegenerate $e^{ \pm}$plasma and photons appears on the left-hand side. The solution of this equation, which essentially coincides with the estimate from Thompson and Duncan (1995)

$$
\tau_{\nu}^{(0)} \simeq \frac{44 \pi^{3}}{175 \zeta(5) C_{+}^{2}} \frac{1}{G_{F}^{2} T^{5}} \simeq 22 \mathrm{~s}\left(\frac{1 \mathrm{MeV}}{T}\right)^{5},
$$

shows that the neutrino cooling time for a fairly weak magnetic field is an order of magnitude shorter than the characteristic duration of a giant flare $\tau_{L T} \simeq(200-400) \mathrm{s}$. Thus, the bulk of the hot-plasma energy is expended in cooling by neutrino emission and such a plasma cannot be the source of an SGR giant flare. Consequently, a mechanism suppressing the neutrino cooling processes is required. As will be shown below, a strong magnetic field that is capable of significantly reducing the hot-plasma energy losses through neutrino emission can act as such a mechanism.

\section{The Case of a Strong Magnetic Field}

In the asymptotic limit of a strong magnetic field, when $e B \gg T^{2} \gtrsim m^{2}$, the emissivity in the electron-positron pair annihilation process (1) is well known and can be represented for a nondegenerate plasma as (Kaminker et al. 1992)

$$
Q_{A}^{(B)}=\frac{\zeta(3) C_{+}^{2}}{48 \pi^{3}} G_{F}^{2} m^{2} e B T^{5}
$$

where $m$ is the electron mass. 
The plasmon decay reaction (2) is also modified significantly by a strong magnetic field, because not only the amplitude of the process but also the dispersion law of plasmon modes change. Calculations show (Kuznetsov et al. 1998) that the emissivity in the strong-field limit is

$$
Q_{P}^{(B)}=\frac{\zeta(5) C_{+}^{2}}{2 \pi^{6}} \alpha G_{F}^{2}(e B)^{2} T^{5}
$$

As we see from this expression, the emissivity in this process can become equal to (11) only at a sufficiently large magnetic field strength $B \simeq 8 \times 10^{15} \mathrm{G}$.

The fusion of two photons (3) in the case of a strong magnetic field was investigated by Rumyantsev and Chistyakov (2008). Since the analytical expression for the neutrino emissivity in this reaction is fairly cumbersome, it is given below in an approximate form that is valid only in the limit $e B \gg T^{2} \gg m^{2}$ :

$$
Q_{\gamma \gamma}^{(B)} \simeq 2.7 \times 10^{18} \frac{\mathrm{erg}}{\mathrm{cm}^{3} \mathrm{~s}}\left(\frac{T}{m}\right)^{9} .
$$

Note that the emissivity in this limit is virtually independent of the magnetic field strength and is lower than that in the annihilation process approximately by three orders of magnitude.

The emissivity in the photoneutrino production process (41) in the limit of a strong magnetic field can be represented as

$$
Q_{F}^{(B)} \simeq \frac{2 C_{+}^{2}}{3 \pi^{6}} \alpha I G_{F}^{2} m^{2} e B T^{5} \ln (T / m),
$$

where the numerical factor I has the following integral representation:

$$
\begin{gathered}
I=\int_{0}^{1} d u \int_{0}^{u} d v \int_{0}^{\infty} \frac{d x x^{4} e^{-2 x}}{e^{x u}+e^{x v}} \times \\
\times\left\{(1-v)\left[(1-v)^{2}-(1-u)^{2}\right] e^{x v}+(1+v)\left[(1+v)^{2}-(1-u)^{2}\right] e^{-x v}\right\} \simeq 0.09 .
\end{gathered}
$$

Comparison of the derived emissivity with (11) shows a negligible contribution from this reaction to the neutrino energy losses of a strongly magnetized nondegenerate plasma.

The neutrino emissivity in the synchrotron emission process (5) in the strong-field limit was obtained by Kaminker and Yakovlev (1993):

$$
Q_{S}^{(B)}=\frac{1-9 / 4 e}{2^{1 / 4} 9 \pi^{9 / 2}} C_{+}^{2} G_{F}^{2}(e B)^{17 / 4} T^{1 / 2} e^{-\sqrt{2 e B} / T} .
$$

As we see from this expression, the emissivity in this limit is exponentially suppressed by the smallness of the number density of electrons and positrons at all Landau levels, except for the ground one. On these grounds, it is generally concluded that the synchrotron emission process cannot play a significant role in the neutrino cooling of a plasma with a strong magnetic field. However, as will be shown below, this conclusion is unjustified.

Assuming that the electron-positron pair annihilation (11), is the dominant plasma neutrino cooling process in the presence of a strong magnetic field, we can find the characteristic neutrino cooling time $\tau_{\nu}^{(B)}$ to a temperature $T$. It can be found from the equation

$$
\frac{d}{d t}\left[\frac{e B}{12} T^{2}\right]=-Q_{A}^{(B)}
$$


where the energy density of an electron-positron plasma whose particles are at the ground Landau level appears on the left-hand side. Solving this equation gives

$$
\tau_{\nu}^{(B)} \simeq \frac{8 \pi^{3}}{3 \zeta(3) C_{+}^{2}} \frac{1}{G_{F}^{2} m^{2} T^{3}} \simeq 760 \mathrm{~s}\left(\frac{1 \mathrm{MeV}}{T}\right)^{3} .
$$

Note that the neutrino cooling time depends only on temperature, because both the plasma energy density and the energy losses through neutrino emission in this limit grow proportionally to the magnetic field strength. It follows from the above estimate that the characteristic neutrino cooling time for a plasma with a strong magnetic field must exceed the giant flare duration $\tau_{L T} \simeq(200-400) \mathrm{s}$ by several times. On these grounds, the authors of the magnetar model concluded that the plasma neutrino emission could be neglected (Thompson and Duncan 1995).

Although the neutrino cooling time in this limit does not depend on magnetic field strength, the neutrino emissivity (11) increases linearly with growing field, reaching the field-free value (6) at $B \simeq 10^{16} G(T / m)^{4}$. Thus, the neutrino emissivity in the annihilation process must be suppressed significantly in a magnetic field whose strength satisfies the following inequality:

$$
4.4 \times 10^{13} G t^{2} \ll B \ll 10^{16} G t^{4}, \text { or } \sqrt{2} \ll x \ll 22 t,
$$

where $t=T / m, x=\sqrt{2 e B} / T$. The lower bound follows from the condition $e B \gg T^{2}$. Note that the commonly assumed magnetic field strength for magnetars $B_{M} \sim 10^{15} \mathrm{G}$ (Duncan and Thompson 1992; Thompson and Duncan 1993) falls within this range at a plasma temperature $T \gtrsim m$ typical of a giant flare. However, the estimate (17) for the neutrino cooling time is valid only if the neutrino emissivity is defined by the asymptotic expression (11). A detailed analysis shows that this expression is applicable only in magnetic fields with strengths

$$
B \gtrsim 7 \times 10^{15} G t^{2}
$$

while the emissivity in this process at smaller strengths can exceed considerably the asymptotic one.

In addition, the neutrino synchrotron emission process (5) is important for plasma cooling even in a strong magnetic field. Indeed, the ratio of the emissivity in this process to the emissivity in the annihilation reaction is given by the expression

$$
\frac{Q_{S}^{(B)}}{Q_{A}^{(B)}}=\frac{\sqrt{2 \pi}}{3 \pi^{2} \zeta(3)}\left(1-\frac{9}{4 e}\right) t^{2} x^{13 / 2} e^{-x},
$$

which has a maximum at $x_{\max }=13 / 2$ equal to $Q_{S}^{(B)} / Q_{A}^{(B)} \simeq 3.5 t^{2}$. Consequently, at a temperature $T \gtrsim m$ typical of a giant flare, the neutrino synchrotron emission process contributes significantly to plasma neutrino cooling in the range of magnetic field strengths (18), where the annihilation process is essentially suppressed.

Thus, our analysis shows that to describe the neutrino cooling of a plasma emitting an SGR giant flare, it is insufficient to consider only the electron-positron pair annihilation (1), because the synchrotron neutrino pair production (5) is a no less important reaction and both these processes make a comparable contribution to the plasma energy losses. In addition, the actual neutrino emissivities in the processes (11) and (5) exceed considerably the asymptotic emissivity (11) in the strong-field limit even in magnetic fields with strengths $B \gtrsim 10^{15}$. 


\section{LOWER BOUND ON THE MAGNETIC FIELD STRENGTH OF A MAGNETAR FROM THE NEUTRINO COOLING RATE}

In this section, the results obtained above are used to model the plasma neutrino cooling for an SGR giant flare. First of all, it should be emphasized that, although such a plasma is fairly hot, it is essentially transparent to neutrinos. Consequently, the neutrinos escape freely from the entire plasma-occupied volume and the energy lost through neutrino emission does not depend on the geometry of the emitting region. To simplify our analysis, we model the fireball as part of a sphere with radius $R_{0}$ whose center is on the magnetar surface (Fig. 1). The distributions of plasma parameters are assumed to be spherically symmetric, just as in the paper by Thompson and Duncan (2001). In our numerical calculations, we used the following temperature and magnetic field strength distributions inside the fireball:

$$
\begin{aligned}
& t(z)=t_{0}(1+z)^{\gamma}, \\
& b(z)=b_{0}(1+z)^{\beta},
\end{aligned}
$$

where $z=r / R_{0}$ is the distance from the fireball center in units of its radius, $t=T / m$ and $b=e B / m^{2}$ are the dimensionless temperature and magnetic field strength, respectively. The parameters of the distributions $t_{0}, b_{0}, \beta$ and $\gamma$ allow the neutrino emission to be described completely. However, in general, we cannot use the results from Thompson and Duncan (2001), where these parameters were obtained by comparing the modeled X-ray emission with the observations of a giant flare from SGR 1900+14, because the neutrino emission was completely excluded in this analysis. Indeed, the plasma at such parameters has only the energy that was observed in this flare as X-ray emission. In contrast, in the case where the energy losses of the medium through neutrino emission are important, the plasma energy must be considerably higher. Thus, the parameters of the distributions (21) and (22) must be found by simultaneously modeling the X-ray emission from the fireball surface and the neutrino cooling of the medium from its volume and by comparing the modeling results with the observed X-ray light curves of SGR giant flares.

Here, we consider a simpler model. The electron-positron plasma is assumed to have an energy $E_{\text {tot }}$ that is expended in cooling by neutrino emission and an SGR X-ray flare with an energy $E_{L T}$. In this case, the energy balance equations can be written as

$$
\begin{gathered}
E_{t o t}=\eta E_{L T}=2 \pi R_{0}^{3} \int_{0}^{1}\left(U_{e^{ \pm}}(z)+U_{\gamma}(z)\right) z^{2} d z, \\
E_{\nu}=(\eta-1) E_{L T}=2 \pi R_{0}^{3} \tau_{L T} \int_{0}^{1} Q_{\nu}(z) z^{2} d z,
\end{gathered}
$$

where $\eta=E_{t o t} / E_{L T}$ the plasma is assumed to be composed of electrons, positrons, and photons, $U_{e^{ \pm}}(z)$ and $U_{\gamma}(z)$ are the local energy densities of these particles, $Q_{\nu}(z)$ is the neutrino emissivity, and the right-hand side of the second equation gives the total energy loss $E_{\nu}$ through neutrino cooling in the giant-flare time $\tau_{L T}$.

\section{Analytical Neutrino Emission Model in the Asymptotic Limit of a Strong Magnetic Field}

As was shown above, the asymptotic expressions for the neutrino emissivities derived in the limit of a strong magnetic field cannot be used to describe the neutrino emission 
of an SGR giant flare. However, we will consider this limiting case, because it admits an analytical solution and provides an insight into the main features of neutrino cooling in the magnetar model. In this case, the neutrino energy losses are determined only by the electron-positron pair annihilation (11):

$$
Q_{\nu}=Q_{A}^{(B)}
$$

whose emissivity is given by Eq. (11). The plasma energy density in this limit is determined by electrons and positrons at the ground Landau level:

$$
U_{e^{ \pm}}+U_{\gamma} \simeq U_{e^{ \pm}} \simeq \frac{m^{4}}{12} b t^{2}
$$

The energy balance equations (23) and (24) in this case can be represented as

$$
\begin{gathered}
\eta E_{L T}=\frac{\pi}{6} J(\beta+2 \gamma) m^{4} R_{0}^{3} b_{0} t_{0}^{2}, \\
(\eta-1) E_{L T}=\frac{\zeta(3) C_{+}^{2}}{24 \pi^{2}} J(\beta+5 \gamma) G_{F}^{2} m^{9} \tau_{L T} R_{0}^{3} b_{0} t_{0}^{5},
\end{gathered}
$$

where the function $J(\delta)$ is defined by the integral

$$
J(\delta)=\int_{0}^{1}(1+z)^{\delta} z^{2} d z=\frac{2^{\delta+1}\left(\delta^{2}+\delta+2\right)-2}{(\delta+1)(\delta+2)(\delta+3)},
$$

and the previously introduced temperature (21) and magnetic field strength (22) distributions are used. The solution of this system of equations can be represented as

$$
\begin{aligned}
& t_{0}(\eta)=\left(\frac{4 \pi^{3}}{\zeta(3) C_{+}^{2}} \frac{J(\beta+2 \gamma)}{J(\beta+5 \gamma)} \frac{1}{G_{F}^{2} m^{5} \tau_{L T}} \frac{\eta-1}{\eta}\right)^{1 / 3} \\
& b_{0}(\eta)=\frac{3\left(\zeta(3) C_{+}^{2}\right)^{1 / 3}}{2^{1 / 3} \pi^{3}} \frac{J^{2 / 3}(\beta+5 \gamma)}{J^{5 / 3}(\beta+2 \gamma)} \frac{G_{F}^{4 / 3} E_{L T} \tau_{L T}^{2 / 3}}{m^{2 / 3} R_{0}^{3}} \frac{\eta^{5 / 3}}{(\eta-1)^{2 / 3}} .
\end{aligned}
$$

Analysis of this solution shows that $t_{0}(\eta)$ and $\beta$ depend very weakly on the exponents $b_{0}(\eta)$ and $\gamma$, respectively. In addition, in the magnetar model (Thompson and Duncan 2001), the change in temperature inside the fireball is assumed to be fairly small from the isothermal case $(\gamma=0)$ to the case of $\gamma=-1$. Of particular interest is $\gamma=-1 / 2$ corresponding to an arbitrary exponent $\beta$. Since a sharp decrease in magnetic field strength in the fireball would lead to excessively intense cooling of its outer layers through neutrino emission, we will assume that the field decreases no faster than the dipolar law $\beta=-3$. Under these assumptions, the following approximate expressions can be used to estimate the temperature $T_{0} \simeq 0.51 \mathrm{MeV} \cdot t_{0}$ and magnetic field strength $B_{0} \simeq 4.4 \times 10^{13} \mathrm{G} \cdot b_{0}$ at the fireball center:

$$
\begin{gathered}
t_{0}(\eta) \simeq 4.4(1-0.6 \gamma) \frac{1}{\tau_{100}^{1 / 3}}\left(\frac{\eta-1}{\eta}\right)^{1 / 3}, \\
b_{0}(\eta) \simeq 2.1\left(1-0.5 \beta+0.3 \beta^{2}\right) \frac{E_{44} \tau_{100}^{2 / 3}}{R_{10}^{3}} \frac{\eta^{5 / 3}}{(\eta-1)^{2 / 3}},
\end{gathered}
$$

where $\tau_{100}=\tau_{L T} / 100 \mathrm{~s}, E_{44}=E_{L T} / 10^{44}$ erg and ${ }_{10}=R_{0} / 10 \mathrm{~km}$. As we see from these expressions, the solution $t_{0}(\eta)$ grows rapidly with plasma energy $E_{t o t}=\eta E_{L T}$ in the 
case of relatively small neutrino energy losses, when $\eta \simeq 1$. Thus, small neutrino energy losses are possible only in a fairly cold plasma. In the region where neutrino cooling dominates, when $\eta \gg 1$, the solution $t_{0}(\eta)$ reaches a constant. The existence of this limiting temperature stems from the fact that the change in fireball temperature through the emission of neutrinos and photons in the flare time $\tau_{L T}$ is neglected in the model under consideration. The solution $b_{0}(\eta)$ contains a divergence at $\eta=1$ which physically corresponds to the impossibility of completely removing the energy losses through neutrino emission. It passes through a minimum at $\eta=5 / 3$ and reaches the asymptotics $\eta$ as $b_{0}(\eta) \sim \eta$ increases further in the range of energies where the neutrino energy losses dominate. Figure 2 presents the corresponding solutions at $\gamma=-1 / 2$ and $\beta=-3$ for the following giant flares:

$$
\begin{array}{ll}
S G R 0526-66: & E_{44}=3.6, \tau_{100}=2.0, \\
S G R 1806-20: & E_{44}=1.3, \tau_{100}=3.8 .
\end{array}
$$

We do not discuss the flare from SGR 1900+14, because it has characteristics at the LT state similar to those of SGR 1806-20.

Let us separately consider another important peculiarity of the solution obtained. If we fix the total plasma energy $E_{t o t}=\eta E_{L T}$ and assume that the temperature $t_{0}>t_{0}(\eta)$ then, in view of Eq. (27), the magnetic field strength $b_{0}$ must be lower than the corresponding solution $b_{0}(\eta)$. It is easy to see that, in this case, the energy losses by such a medium $E_{\nu}$ through neutrino emission that are defined by the right-hand side of Eq. (28) will increase, i.e., they will become greater than $\left(E_{t o t}-E_{L T}\right)$. In contrast, for $t_{0}<t_{0}(\eta)$ the value of $b_{0}$ must be higher than $b_{0}(\eta)$ and the neutrino energy losses will decrease. Thus, the solution (29) defines the maximum temperature, while the solution (30) defines the minimum magnetic field strength at which the neutrino energy losses leave an energy in the plasma no less than $E_{L T}$ observed in the photon emission. In addition, since $t_{0}(\eta)$ has a global maximum and $b_{0}(\eta)$ has a global minimum, the upper and lower bounds, respectively, on the admissible temperature and the magnetic field strength at the fireball center correspond to them:

$$
\begin{aligned}
& T_{0}^{(\max )} \simeq 2.2 \mathrm{MeV}(1-0.6 \gamma) \frac{1}{\tau_{100}^{1 / 3}} \\
& B_{0}^{(\min )} \simeq 2.8 \times 10^{14} G\left(1-0.5 \beta+0.3 \beta^{2}\right) \frac{E_{44} \tau_{100}^{2 / 3}}{R_{10}^{3}},
\end{aligned}
$$

They are needed for the energy emitted by the plasma in photons to be no less than $E_{L T}$. As was noted above, the existence of a maximum temperature of the medium stems from the fact that its change through the emission of photons and neutrinos in the flare time $\tau_{L T}$ is disregarded in the model under consideration. It follows from the condition (36) that, depending on the magnetic field strength, a plasma with an arbitrary energy $E_{\text {tot }}$ can emit an energy in photons no greater than

$$
E_{L T}^{(\max )} \simeq 10^{44} \operatorname{erg} \frac{B_{15} R_{10}^{3}}{\tau_{100}^{2 / 3}},
$$

where $B_{15}=B_{0} / 10^{15} G$ and $\beta=-3$. As we see from this expression, the fireball radius $R_{0}$ is the most important parameter defining the admissible energy release in photons, while the reasonable energy release itself is close to the typical energy of SGR giant flares. 
It should be noted that a modulation of the emission intensity by the neutron star spin period (Mazets et al. 1979; Ibrahim et al. 2001; Mereghetti et al. 2005; Frederiks et al. 2007) is clearly traceable for all of the known SGR giant flares at the LT stage. The presence of such pulsations leads to the conclusion that the size $R_{0}$ of the plasma-occupied region must be close to the neutron star radius $R_{N S}$. Under this assumption and for a standard value of $R_{N S} \simeq 10 \mathrm{~km}$ and a typical energy $E_{L T} \sim 10^{44} \mathrm{erg}$ of an SGR giant flare at the LT stage, the magnetic field strength must be $B_{0} \gtrsim 10^{15} \mathrm{G}$. It is interesting to compare this value with the upper bound on the magnetic field strength that follows from an estimate of the magnetic dipole losses for a magnetar:

$$
B_{M D} \simeq 2.1 \times 10^{15} G \frac{1}{\sin \theta} \frac{M_{1.4}^{1 / 2}}{\widetilde{R}_{10}^{2}}\left(P_{10} \dot{P}_{-10}\right)^{1 / 2} .
$$

Here, $M_{1.4}=M_{N S} / 1.4 M_{\odot}, \widetilde{R}_{10}=R_{N S} / 10 k m, P_{10}=P / 10 s, \dot{P}_{-10}=\dot{P} / 10^{-10}$, where $M_{N S}, P$, and $\dot{P}$ are the neutron star mass, period, and spindown rate, respectively, $\theta$ is the angle between the angular velocity and magnetic moment vectors. It is easy to see that these bounds almost coincide at $R_{0} \simeq R_{N S}$. Thus, the above estimates leave open only a narrow range of magnetar magnetic field strengths even in the simplified model that grossly underestimates the energy losses of the medium through neutrino emission and, consequently, the minimum magnetic field strength $B_{0}^{(m i n)}$.

\section{Modeling the Neutrino Cooling of SGR 0526-66 and SGR 1806-20}

In the previous section, we studied an analytical model for the neutrino cooling of a plasma producing an SGR giant flare that is based on the asymptotic expressions for the plasma energy density and neutrino emissivity derived in the limit of very strong magnetic fields. In this section, we will use the expressions valid for an arbitrary magnetic field strength for a more realistic modeling of the neutrino cooling process.

Since the photon mean free path in the plasma under consideration is small, the electrons, positrons, and photons are in local thermodynamic equilibrium and, hence, the equilibrium distribution functions can be used to describe them. In this case, the plasma energy density will be defined by the expressions

$$
\begin{gathered}
U_{\gamma}=\frac{\pi^{2} m^{4}}{15} t^{4}, \\
U_{e^{ \pm}}=\frac{m^{4}}{\pi^{2}} b\left[\int_{0}^{\infty} \frac{\left(x^{2}+1\right)^{1 / 2}}{e^{\sqrt{x^{2}+1} / t}+1} d x+2 \sum_{n=1}^{\infty} \int_{0}^{\infty} \frac{\left(x^{2}+2 b n+1\right)^{1 / 2}}{e^{\sqrt{x^{2}+2 b n+1} / t}+1} d x\right] .
\end{gathered}
$$

As was shown above, the electron-positron pair annihilation (11) and the neutrino synchrotron emission (5) are the main neutrino cooling processes for the plasma under consideration:

$$
Q_{\nu}=Q_{A}+Q_{S}
$$

Here, for the neutrino emissivity of the annihilation process $Q_{A}$, we used the interpolation 
formula (Kaminker et al. 1992)

$$
\begin{aligned}
& Q_{A}=\frac{G_{F}^{2} m^{9}}{\pi^{4}}\{ {\left[\left(C_{+}^{2}+C_{-}^{2}\right)\left(\frac{1}{2}+\frac{15}{8} t\right) t^{3}+C_{+}^{2} P(t)\right] F(t, b) } \\
&\left.+\left[C_{+}^{2}+C_{-}^{2}+\left(C_{+}^{2}-C_{-}^{2}\right) \frac{b}{1+b}\right] \frac{b^{2} S(t)}{12(1+b)}\right\} \exp \left(-\frac{2}{t}\right), \\
& P(t)=t^{4}\left(1+3.581 t+39.64 t^{2}+24.43 t^{3}+36.49 t^{4}+18.75 t^{5}\right), \\
& S(t)=t\left(1+1.058 t+0.6701 t^{2}+0.9143 t^{3}+0.472 t^{4}\right), \\
& F(t, b)=\frac{1}{R_{1} R_{2} R_{3}}, \quad R_{i}=1+c_{i} \frac{b}{t^{2}} \exp \left(\frac{\sqrt{2 b}}{3 t}\right),
\end{aligned}
$$

where $C_{+}^{2} \simeq 1.675, C_{-}^{2} \simeq 0.175$ the constants $c_{1} \simeq 3.106 \times 10^{-6}, c_{2} \simeq 1.491 \times 10^{-3}$, $c_{3} \simeq 4.839 \times 10^{-6}$. For the emissivity of the synchrotron neutrino pair production reaction, we used the interpolation expression from Kaminker and Yakovlev (1993)

$$
\begin{gathered}
Q_{S}=\frac{G_{F}^{2} m^{9}}{120 \pi^{3}} N_{e^{ \pm}}\left(C_{+}^{2} F_{+}-C_{-}^{2} F_{-}\right), \\
N_{e^{ \pm}}=\frac{2 m^{3}}{\pi^{2}} b \sum_{n=1}^{\infty} \int_{0}^{\infty} \frac{d x}{e^{\sqrt{x^{2}+2 b n+1} / t}+1}, \\
F_{+}=\frac{b^{6}}{\left(1+a_{1} b\right)^{3}}\left(1+\frac{a_{2} t}{\left(1+a_{1} b\right)^{1 / 2}}\right)^{6}\left(1+\frac{a_{3} t b}{\left(1+a_{1} b\right)^{3 / 2}}\right)^{-5}\left[1+\ln \left(1+\frac{a_{4} t b}{\left(1+a_{5} b\right)^{3 / 2}}\right)\right], \\
F_{-}=\frac{b^{6}\left(1+a_{6} t\right)^{6}}{\left(1+a_{1} b\right)^{6}}\left(1+\frac{a_{3} t b}{\left(1+a_{1} b\right)^{3 / 2}}\right)^{-5},
\end{gathered}
$$

where $N_{e^{ \pm}}$is the number density of electrons and positrons at all Landau levels, except for the ground one. Here, the constants $a_{1} \simeq 0.955, a_{2} \simeq 9.439, a_{3} \simeq 23.31, a_{4} \simeq 0.26$, $a_{5} \simeq 0.168, a_{6} \simeq 0.971$.

We modeled neutrino cooling for giant flares from SGR 0526-66 and SGR 1806-20 with the characteristics (33) and (34). As was noted above, the SGR 1900+14 flare at the LT stage is similar in its properties to SGR 1806-20 and below we do not separate them. Our numerical calculations confirm all of the qualitative conclusions reached in the previous section for the analytical model of neutrino cooling, but the numerical values of the plasma temperature and magnetic field strength change significantly. Below, we present the results for the case of $\beta=-3$ which corresponds to a dipole magnetic field configuration in the fireball. The law of change in temperature was chosen with an exponent characteristic of the magnetar model, $\gamma=-1 / 2$ which was compared with the isothermal case of $\gamma=0$. The fireball radius was chosen to be $R_{0}=10 \mathrm{~km}$, which corresponds to the standard radius of a neutron star. The results of our numerical solution of the system of equations (23, (24) for the SGR giant flares under consideration are presented in Figs. 3 and 4. As we see from the plots, the general trend in the behavior of the temperature and magnetic field strength as a function of the total plasma energy $E_{t o t}=\eta E_{L T}$ is the same as that in the analytical model. However, the plasma being analyzed turns out to be colder and the magnetic field strength in it must be higher by several times. Note that the dependence of the solutions on parameters $\gamma$ and $\beta$ also remains similar to the analytical model (see Eqs.(31) and (32)). Thus, the calculated magnetic field strength in the fireball is virtually independent of the law of change in 
temperature, as is demonstrated in Fig. 4. We emphasize once again that the derived field strength at fixed total plasma energy is minimally possible for the emission of the observed giant-flare energy $E_{L T}$. Note also that the global minimum of the magnetic field strength for the SGR giant flares in question takes place at $\eta \simeq 1.3$ instead of $\eta=5 / 3$ in the analytical model and, hence, the energy losses through neutrino emission are reduced approximately by half. However, the losses remain fairly large and their reduction requires a great increase in magnetic field strength even in this case.

We separately investigated the dependence of the solutions obtained on the plasmaoccupied fireball radius $R_{0}$. In contrast to the analytical model where the plasma temperature did not depend on this parameter but was determined only by the giant-flare duration, our numerical calculation showed that the temperature of the plasma-occupied region decreases with its increasing sizes. The dependence of the magnetic field strength on $R_{0}$ remains similar to the analytical model, but the law $b_{0} \sim R_{10}^{-3}$ is replaced by a slightly faster decrease in strength. Figure 5 presents the numerically calculated global minimum of themagnetic field strength $b_{\min } \equiv b_{0}^{(\min )}(\eta)$ at $R_{0}=5,10$ and $15 \mathrm{~km}$. It is well fitted by the formulas

$$
S G R 0526-66: \quad b_{\min } \simeq 220 R_{10}^{-3}+140 ; \quad S G R 1806-20: \quad b_{\min } \simeq 120 R_{10}^{-3}+60,
$$

which are valid both for the isothermal case $(\gamma=0)$ and for the case of $\gamma=-1 / 2$. As we see from the plots, the minimum magnetic field strength near $R_{0} \simeq 5 \mathrm{~km}$ exceeds considerably $B_{0}^{(\min )} \sim 10^{16} \mathrm{G}$. Therefore, the situation where the plasma occupies a fairly extended region $R_{0} \gtrsim 10 \mathrm{~km}$ is of greatest interest in our analysis. In this case, the minimum magnetic field strength at the fireball center and the upper bound on the field strength obtained from our estimate of the magnetar magnetic dipole losses can be represented as

$$
\begin{array}{lll}
S G R 0526-66: & B_{0}^{(\min )} \simeq 2 R_{10}^{-3} \times 10^{16} G, & B_{M D} \simeq 2 \widetilde{R}_{10}^{-2} \times 10^{15} G ; \\
S G R 1806-20: & B_{0}^{(\min )} \simeq R_{10}^{-3} \times 10^{16} G, & B_{M D} \simeq(2-6) \widetilde{R}_{10}^{-2} \times 10^{15} G ; \\
S G R 1900+14: & B_{0}^{(\min )} \simeq R_{10}^{-3} \times 10^{16} G, & B_{M D} \simeq(2-3) \widetilde{R}_{10}^{-2} \times 10^{15} G,
\end{array}
$$

where $B_{0}^{(\min )}$ for SGR $1900+14$ is the same as that for the SGR 1806-20 giant flare. To estimate the strength $B_{M D}$, we used the parameters $M_{N S}=1.4 M_{\odot}$ and $\theta=\pi / 4$ and the following periods and spindown rates: SGR 0526-66: $P_{10} \simeq 0.81, \dot{P}_{-10} \simeq 0.65$; SGR 180620: $P_{10} \simeq 0.756, \dot{P}_{-10} \simeq 0.8 \div 8 ;$ SGR $1900+14: P_{10} \simeq 0.515, \dot{P}_{-10} \simeq 0.5 \div 1.4$, (Mereghetti 2008). As was discussed above, the modulation of the X-ray intensity for known flares by the magnetar spin period leads to the conclusion that the fireball radius $R_{0}$ cannot differ significantly from the neutron star radius $R_{N S}$. As follows from the estimates (44) - (46), under the condition $R_{0} \simeq R_{N S}$ the upper bound on the magnetic field strength of the magnetars under consideration turns out to be lower than the minimally possible value required for the suppression of neutrino emission.

\section{CONCLUSIONS}

In this paper, we estimated the energy losses of a nondegenerate relativistic $(T \gtrsim m)$ electron-positron plasma through neutrino emission based on the magnetar model of an SGR giant flare (Thompson and Duncan 1995, 2001). In the absence of a magnetic field, the plasma energy losses through neutrino emission were shown to be too large to provide the observed energy release at the LT stage of an SGR giant flare. It follows 
from our analysis of the neutrino processes considered that in the case of a strongly magnetized plasma $e B \gg m^{2}$ important for the magnetar model, not only the electronpositron annihilation into a neutrino pair (1) but also the neutrino synchrotron emission process (5), which is usually neglected, make a major contribution to the neutrino energy losses. The plasma neutrino emissivities in these processes were shown to be significant even in the case of fairly strong magnetic fields $B \gtrsim 10^{15} \mathrm{G}$. Thus, when the X-ray emission of an SGR giant flare is modeled, the plasma energy losses through neutrino emission should be properly taken into account, which was not done by Thompson and Duncan $(1995,2001)$.

To investigate the main features of the fireball neutrino cooling, we considered a simple analytical model. It clearly shows that the photon emission at an arbitrary plasma energy cannot exceed some maximum value dependent on the size of the plasma occupied region and the magnetic field strength in the plasma. Thus, the minimally possible magnetic field strength of a magnetar that provides sufficient suppression of its neutrino emission at the long tail stage can be found for the observed energy of a giant flare. We numerically modeled the neutrino cooling of the SGR 0526-66, SGR 1806-20, and SGR 1900+14 giant flares, including all of the neutrino reactions important for this process. The lower bound on the magnetic field of these objects corresponding to the energy observed in photons at the LT stage of SGR giant flares was shown to disagree with the upper bound from our estimate of their magnetic dipole losses. Consequently, the magnetar model of an SGR giant flare considered here cannot provide the energetics observed at the long tail stage in a fairly wide range of parameters.

We modeled neutrino cooling under the simplifying assumptions that the fireball temperature and sizes did not change in the time of an SGR giant flare. Allowance for the evolution of these characteristics must lead to a reduction in the energy losses of the medium through neutrino emission compared to the model considered. However, it is hard to expect that the minimally possible magnetic field strength required for the suppression of the fireball neutrino emission can be significantly lower than the estimates obtained here and can become equal to the upper bounds following from our estimate of the magnetic dipole losses for magnetars. Note also that the magnetar model is not without other contradictions either. In particular, an important problem (Malov and Machabeli 2006) that arises in attempting to explain the existence of radio emission detected from SGRs and AXPs (Malofeev et al. 2005) should be pointed out.

In conclusion, note that the magnetar model considered here was further developed by Lyutikov (2006), Beloborodov and Thompson (2007), and Beloborodov (2009). These authors used the more realistic magnetohydrodynamic approach to describe the magnetar corona and revealed the effect of additional plasma heating due to the magnetic field energy. Using this approach to describe SGR giant flares at the LT stage could partially solve the problem of energy deficiency discussed here.

\section{ACKNOWLEDGMENTS}

We wish to thank G.S. Bisnovatyi-Kogan, S.I. Blinnikov, and N.V.Mikheev for constant interest in the work and fruitful discussions of the results. We are grateful to A.D.Kaminker, I.F.Malov, S.B. Popov, D.A. Rumyantsev, M.V. Chistyakov, A.I. Tsygan, and D.G. Yakovlev for helpful discussions and valuable remarks. We are also grateful to the referee for the remarks whose allowance improved the paper. The study was supported by the Scientific and Scientific-Pedagogical Personnel of Innovational Russia Federal Goal-Oriented Program for 2009-2013 (State contract no. P2323) and, in part, 
by the Development of the Scientific Potential of Higher School Program of the Ministry of Education and Science of the Russian Federation (project no. 2.1.1/510).

\section{References}

[1] G. Beaudet, V. Petrosian, and E. E. Salpeter, Astrophys. J. 150, 979 (1967).

[2] A. M. Beloborodov, Astrophys. J. 703, 1044 (2009).

[3] A. M. Beloborodov and C. Thompson, Astrophys. J. 657, 967 (2007).

[4] G. S. Bisnovatyi-Kogan,Fiz. Elem. Chastits At. Yadra 37, 1235 (2006) [Phys. Part. Nucl. 37, 647 (2006)].

[5] R. Dib, V.M. Kaspi, F.P. Gavriil et. al., Astrophys. J. 673, 1044 (2008).

[6] R.C. Duncan, C. Thompson, Astrophys. J. 392, L9 (1992).

[7] D. D. Frederiks, S. V. Golenetskii, V. D. Palshin, et al., Pis'ma Astron. Zh. 33, 3 (2007) [Astron. Lett. 33, 1 (2007)].

[8] E. Gogus, P. M.Woods, C. Kouveliotou, et. al., Astrophys. J. 532, L121 (2000).

[9] A.I. Ibragim, T.E. Strohmayer, P.M. Woods et. al., Astrophys. J. 558, 237 (2001)

[10] N. Itoh, H. Hayashi, A. Nishikawa et. al., Astrophys. J. 102, 411 (1996)

[11] A. D. Kaminker and D. G. Yakovlev, Zh. Eksp. Teor. Fiz. 103, 438 (1993) [J. Exp. Theor. Phys. 76, 229 (1993)].

[12] A.D. Kaminker, O.Yu. Gnedin, D.G. Yakovlev et. al., Phys. Rev. D 46, 4133 (1992).

[13] E. M. Kantor and M. E. Gusakov, Mon. Not. R. Astron. Soc. 381, 1702 (2007).

[14] A.V. Kuznetsov, N.V. Mikheev, L.A. Vassilevskaya, Phys. Lett. B 427, 105 (1998).

[15] M. Lyutikov, Mon. Not. R. Astron. Soc. 367, 1594 (2006).

[16] V.M. Malofeev, I.F. Malov, D.A. Teplych et.al., Astron. Reports 49, 242 (2005).

[17] I.F. Malov, G.Z. Machabeli, Astron. and Astrophys. Transaction 25, 7 (2006).

[18] E.P. Mazets, S.V. Golentskii, V.N. Ilinskii et. al., Nature 282, 587 (1979).

[19] S. Mereghetti, Astron. Astrophys., 15, 225 (2008).

[20] S. Mereghetti, D. Gotz, A. von Keinlin et. al., Astrophys. J. 624, L105 (2005).

[21] D. A. Rumyantsev and M. V. Chistyakov, Zh. Eksp. Teor. Fiz. 134, 627 (2008). [J. Exp. Theor. Phys. 107, 533 (2008)].

[22] T.E. Strohmayer, A.L. Watts, Astrophys. J. 653, 593 (2006).

[23] C. Thompson, R.C. Duncan, Astrophys. J. 408, 194 (1993).

[24] C. Thompson, R.C. Duncan, Mon. Not. R. 275, 255 (1995). 
[25] C. Thompson, R.C. Duncan, Astrophys. J. 473, 322 (1996).

[26] C. Thompson, R.C. Duncan, Astrophys. J. 561, 980 (2001).

[27] A.L. Watts, T.E. Strohmayer, Astrophys. Space Sci. 308, 625 (2007).

[28] P. M. Woods, C. Kouveliotou, E. Gogus, et al., Astrophys. J 629, 985 (2005).

[29] D.G. Yakovlev, A.D. Kaminker, O.Y. Gnedin, P. Haensel, Phys. Rept. 354, 1 (2001). 


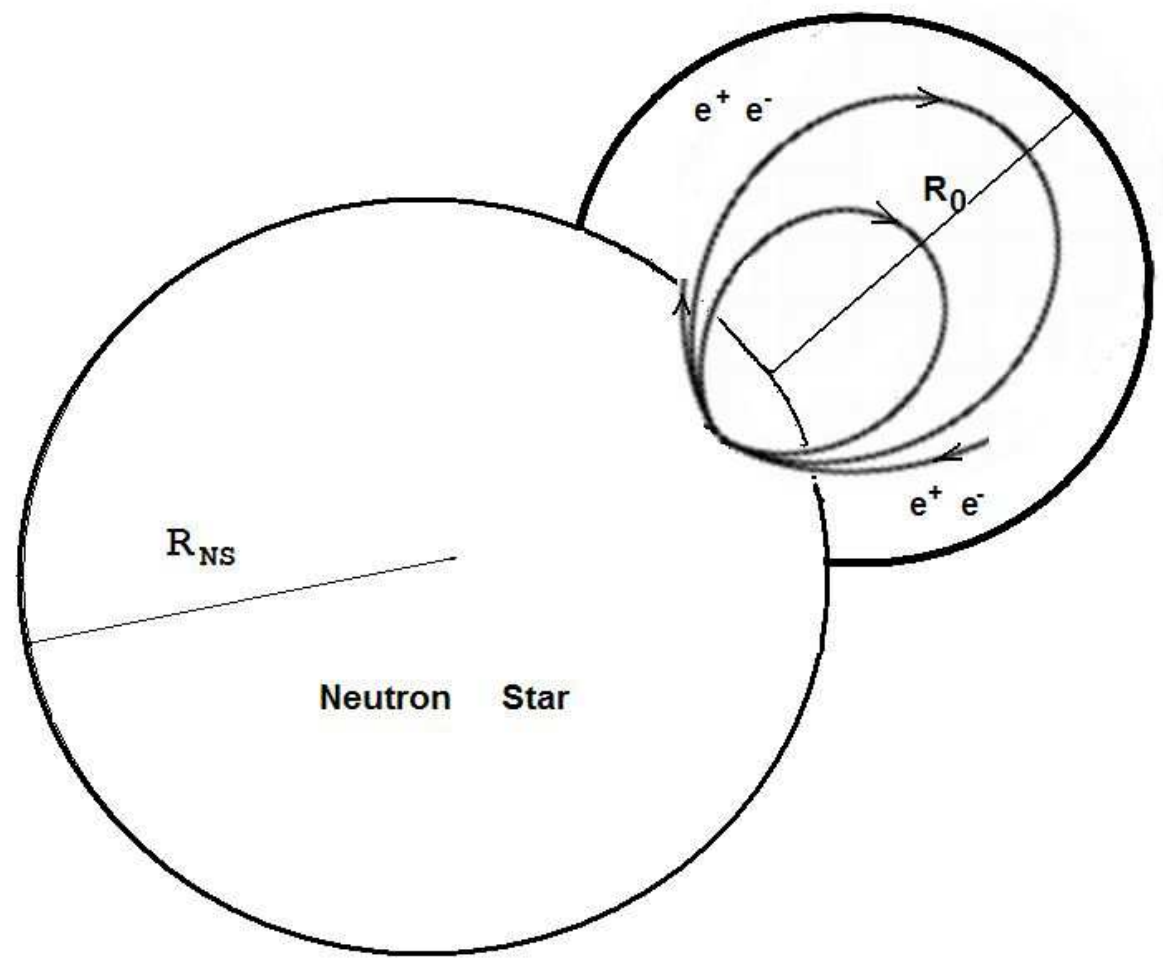

Figure 1: Scheme of the region of a ball with radius $R_{0}$ filled with an electron-positron plasma and trapped by a poloidal magnetic field with field lines closed on the magnetar crust. 

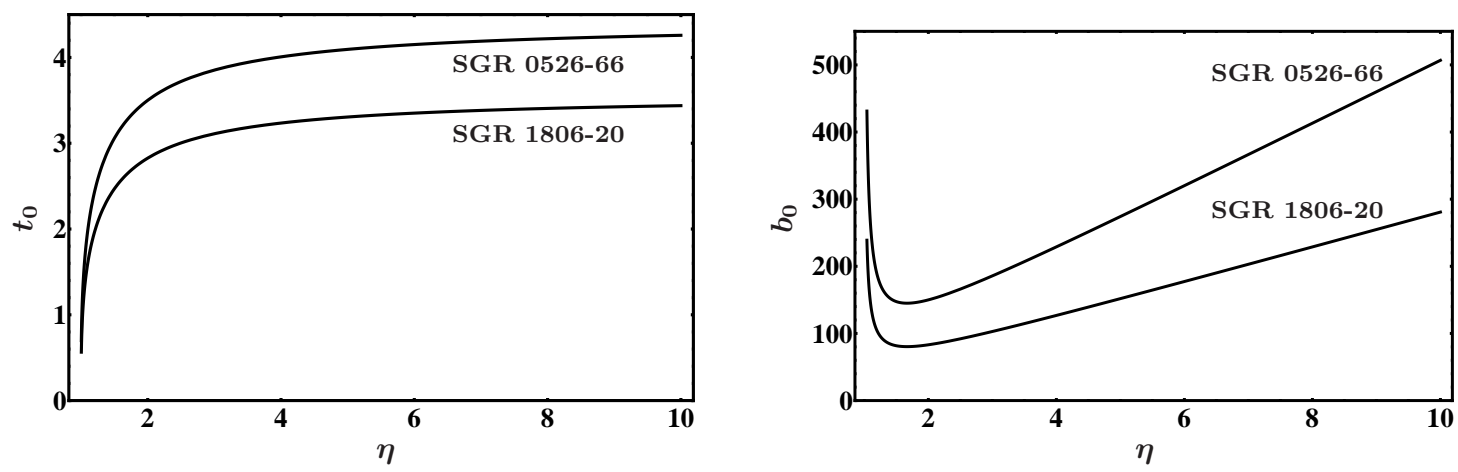

Figure 2: Dependences of the temperature $t_{0}(a)$ and magnetic field strength $b_{0}(b)$ at the fireball center on parameter corresponding to the analytical solution. The lines are drawn for $R_{0}=10 \mathrm{~km}$ and the parameters $\gamma=-1 / 2$ and $\beta=-3$.
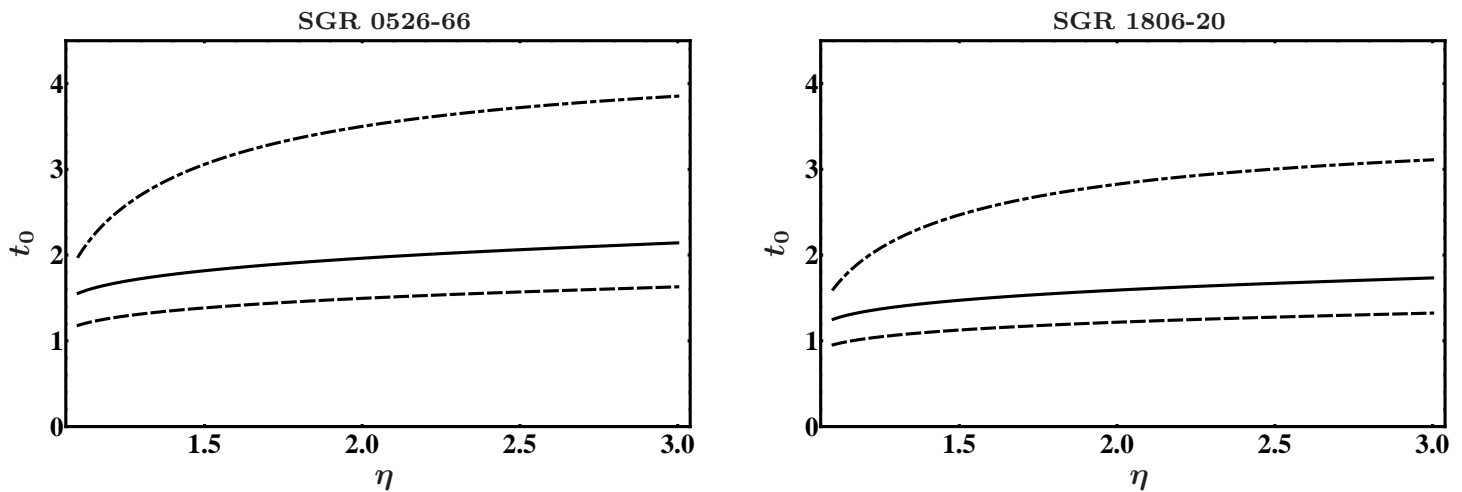

Figure 3: Temperature $t_{0}$ at the fireball center versus parameter for the SGR 052666 (a) and SGR 1806-20 (b) flares at $R_{0}=10 \mathrm{~km}$ and $\beta=-3$. The solid, dashed, and dashdotted lines correspond to $\gamma=-1 / 2, \gamma=0$, and the analytical solution at $\gamma=-1 / 2$, respectively.
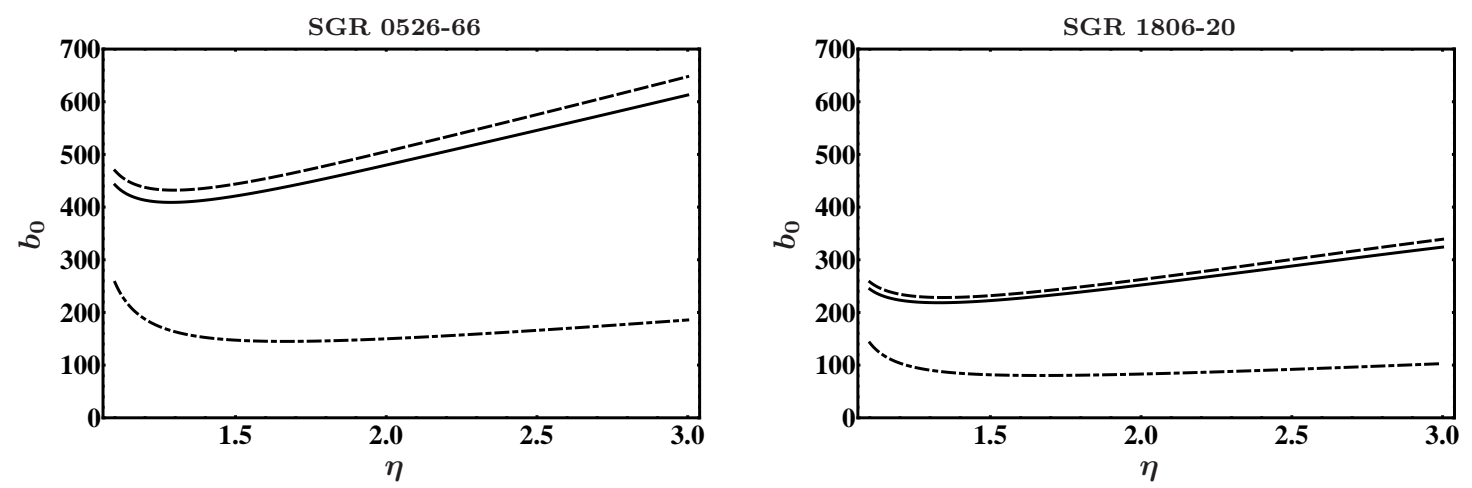

Figure 4: Magnetic field strength b0 at the fireball center versus parameter for the SGR 0526-66 (a) and SGR 1806-20 (b) flares at $R_{0}=10 \mathrm{~km}$ and $\beta=-3$. The solid, dashed, and dashdotted lines correspond to $\gamma=-1 / 2, \gamma=0$, and the analytical solution at $\gamma=-1 / 2$, respectively. 

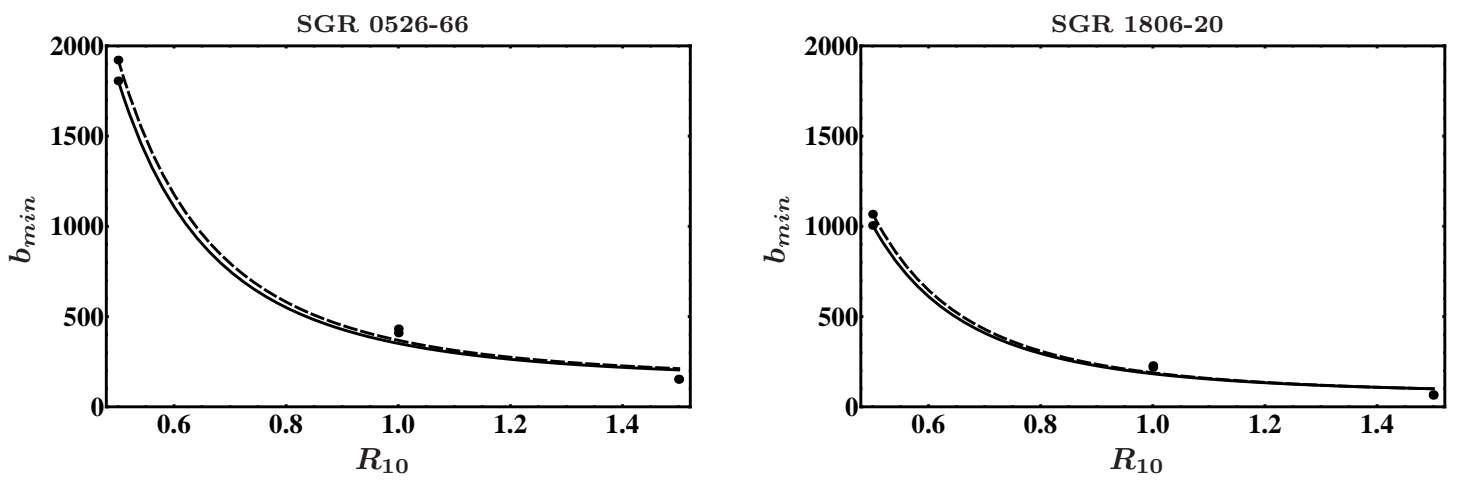

Figure 5: Minimum magnetic field strength $b_{0}^{\min }$ versus radius $R_{10}$ of the plasma-occupied region for the SGR 0526-66 (a) and SGR 1806-20 (b) flares. The filled circles correspond to the calculated values of $b_{0}^{\text {min }}$ at $R_{0}=5,10,15 \mathrm{~km}$ and $\gamma=-1 / 2, \beta=0$. The solid and dashed lines represent the fits to this dependence at $\gamma=-1 / 2$ and $\gamma=0$, respectively. 